\title{
Pensar epistémico y político desde la perspectiva del sujeto
}

\section{Epistemological and political thinking from the perspective of the subject}

\author{
doi: http://dx.doi.org/10.32870/ \\ espiral.v23i66.5297
}

\begin{abstract}
Resumen
Explicitar los posicionamientos epistémicos y ético-políticos es necesario para reconocer la materia de trabajo que es producir conocimiento acerca de lo social. Dar cuenta de su implicación es el objetivo de este artículo, lo cual mostramos al plantear la comunidad de aprendizaje como analizadora de los intentos por remontar la fetichización del proceso de investigación/ enseñanza-aprendizaje, metodología que complementamos con la crítica y la problematización en cuanto forma de reflexión, de tal manera que nos hacemos parte de la renovación del conocimiento socialhistórico, lo que sucede por dejar de pensar desde la fragmentación disciplinar y situar en el centro al sujeto.
\end{abstract}

Palabras clave: pensamiento crítico, universidad, comunidad de aprendizaje, epistemología y autonomía.
Rafael Sandoval Álvarez*

Rocío Salcido Serrano*•

\begin{abstract}
To explain the epistemics and ethic-politics positioning is necessary for to recognize the matter of the work that is to produce knowledge about the social. This article looks for its implication, which is showed setting the community of knowledge like analyzer of the intents for to assume the fetishization of the research/teaching-learning process. This methodology is complimented with the critics and problematization like a reflection form, so we are part of the renovation or the socio-historical knowledge, which happens for stop thinking from the disciplinary fragmentation and for put in the center to the subject.
\end{abstract}

Keywords: Critical thinking, university, learning community, epistemology and autonomy.

\footnotetext{
- Profesor-Investigador del Instituto Nacional de Antropología e Historia (INAH) y Profesor Docente del Departamento de Sociología del Centro Universitario de Ciencias Sociales y Humanidades, Universidad de Guadalajara, México.—rafaelsandoval57@yahoo.com - Profesora-Investigadora del Departamento de Filosofía del Centro Universitario de Ciencias Sociales y Humanidades, Universidad de Guadalajara, México.
} 


\section{Introducción'}

En toda forma de investigar y de problematizar está la exigencia de reflexividad, que deviene de las conciencias histórica y epistémica, indispensables para reconocer el lugar desde donde se realiza la labor de investigación, la razón por la que se hace y contra quién se hace. Ambas formas de conciencia son condición para el reconocimiento de las potencialidades y lo inédito de la realidad; los aspectos anteriores se tornan profundamente contradictorios cuando se enfrentan en el ámbito universitario, porque, no podemos obviarlo, las universidades institucionalmente han orientado sus intereses y objetivos hacia la sobrevivencia de sí mismas en el marco de sociedades capitalistas. Aunque la magnitud de la cuestión desborda con mucho lo que podemos desarrollar en un artículo, damos cuenta de algunos de los elementos prefigurantes de una experiencia ambigua respecto de su forma, pero no en relación con su intención y sentido. A ello nos avocaremos en este artículo.

Los sujetos sociales están como cuestión central en la producción social del conocimiento. Por otro lado, es necesario reconocer que el conocimiento tiene también su valor de uso y su valor de cambio, como cualquier otra producción en el sistema capitalista. Por tanto, las contradicciones se potencian, por ejemplo, al entrar en tensión con la intención y el límite del proceso de investigación, pues, si queremos ir más allá de la relación capitalista, ponderar el valor de uso del conocimiento en ese contexto de producción social de sujetos es una fuente de cuestionamientos a las prácticas de los investigadores, haciendo manifiesto el antagonismo y

I. Este artículo es producto del proyecto colectivo en curso Cómo pensar la revolución hoy, el hacer y el pensar político zapatista (2013-2016), adscrito al Centro Universitario de Ciencias Sociales y Humanidades de la Universidad de Guadalajara, propuesto por R. Salcido, R. Sandoval y M. Gallegos para abordar el pensamiento político concreto y la labor reflexiva de los sujetos de los movimientos de resistencia en sus formas de hacer, su lenguaje y dimensión utópica.

\section{0}


la contradicción que surgen al desplegar un hacer con una pretensión de sentido no capitalista a la que subyace una subjetividad como la que reivindica la autonomía, aunque ella misma no es subjetividad autónoma totalmente. A propósito de ello, la perspectiva del sujeto no significa sólo orientación cognitiva, sino también episteme y, como factor crítico, formas de hacer, unas donde se reconoce el antagonismo social, se problematiza y cuestiona la perspectiva dominante de la relación social capitalista a la que le es funcional el conocimiento producido.

Por eso, nombrar a nuestra orientación como un pensar de lo concreto desde la perspectiva del sujeto es nuestro modo de enunciar el desafío en la generación de conocimiento pertinente para el despliegue de procesos sociales desde y para los sujetos, lo que no significa que se deba hablar de aspectos específicos cada vez, prácticas singulares, mucho menos si tratamos la dimensión epistemológica, sino que esto involucra la necesidad de conciencia histórica y política en los profesores e investigadores que asumimos, a modo de horizonte, la emancipación y la autonomía. Esto hace de la exigencia de colocarse ante y contra la fetichización del proceso de enseñanza-aprendizaje y la alienación del sujeto que lo produce una condición del trabajo de investigación y de hacer política; este desafío lo enfrentamos en el intento por forjar una comunidad de aprendizaje conformada por sujetos autónomos, plagada de contradicciones porque se realiza a contracorriente de las tendencias que propician y se basan, por ejemplo, en la separación sujeto-objeto, el distanciamiento reflexivo, la objetividad valorativa, entre otros.

En este sentido, para nosotros, uno de los aspectos problemáticos es la apropiación de la materia de trabajo, no entendida en cuanto recuperación y defensa de derechos laborales, sino como compromiso con la labor y sus implicaciones hacia los sujetos sociales. De igual forma, este trabajo nos ha implicado reconsiderar la idea de realidad como algo dado, 
para pensarla como procesos creados y articulados por los sujetos, bajo la idea de producir conocimiento en perspectiva transdisciplinar y situados en el horizonte de las formas de hacer y transformar la sociedad, ${ }^{2}$ reconociendo que la historia se ha hecho desde la cotidianidad, cuyo significado es que no hay historia ni proyecto sin sujeto; por ende, precisamos ética y políticamente pensar desde el sujeto generador de cultura. ${ }^{3}$

En nuestra experiencia, ha sido la comunidad de aprendizaje, comprendida como ensayo de formación con una epistemología crítica y una ética de la autonomía, ${ }^{4}$ la que nos ha llevado a plantearnos preguntas acerca de lo que se podría llegar a ser relativas, por consecuencia, a las posibilidades de des-subjetivación de quienes instituyen la universidad y su resubjetivación con base en las significaciones de la autonomía y la dignidad. A propósito de las posibilidades de instituir la comunidad de aprendizaje desde el trabajo cotidiano de la docencia y la compartición del trabajo de investigación, también nos preguntamos ¿de qué manera las formas

2. Estamos conscientes de las limitaciones que representan las disciplinas cuando se plantea generar conocimiento socialmente pertinente. Al respecto, las perspectivas y orientaciones son diversas. Aquí aspiramos a mostrar algo de lo que constituye la manera en que enfrentamos la cuestión sin que sea el objetivo exponer un mapa completo de los aspectos problemáticos implicados.

3. La reflexión y el referente del que partimos es el reconocimiento de la realidad de sujetos que luchan y resisten a la dominación capitalista, de la historia de la resistencia como la premisa epistémica y ética fundamental, cuya exigencia es reconocer que hacer política construye lo colectivo entre las personas, en tanto se haga en función de la satisfacción de la necesidad y la realización del deseo, con el límite de no lastimar al otro. Esta forma de hacer política instituye la solidaridad y el respeto a la diferencia, entendida como reconocimiento de la alteridad y la autonomía del otro.

4. Entendemos por autonomía un proyecto y ruptura de la heteronomía, sea esta provocada por sujetos individuales o colectivos, pero siempre en el entendido de que no puede haber autonomía individual sin el respeto de la autonomía colectiva y viceversa, que deviene como movimiento histórico de los sujetos por arribar a la autoinstitución lúcida de la sociedad. El sentido último de la autonomía es darse la propia ley y autolimitarse, lo cual implica interrogar y poner en tela de juicio las propias instituciones, quitarles el carácter de incuestionables y asumir que son los sujetos quienes les confieren a las instituciones el poder que tienen. La idea de autonomía es antagónica a las formas de autoritarismo. 
de hacer de la pluralidad de sujetos de la comunidad de aprendizaje se corresponden con la apropiación del espacio de aprendizaje desde posiciones de autonomía y dignidad?

Esas interrogantes nos han llevado a asumir el reto de tornarnos sujetos en y del proceso de enseñanza-aprendizaje, de investigación y difusión. De ello presentamos dos aspectos implicados en el ejercicio de resubjetivación mencionado antes: 1) investigar y formarse a contracorriente de la racionalidad instrumental; y 2) la comunidad de aprendizaje en cuanto modo de hacer, que también expresa la aspiración respecto de nuestro quehacer en el marco de la institución universitaria. En conjunto, a esto lo conceptuamos como un posicionamiento antagónico en un marco de cierre heterónomo del espacio universitario.

\section{Contexto en el que emergen quehaceres y prácticas antagónicas}

Preceden a la clausura de la universidad el surgimiento de las disciplinas y la consecuente fragmentación del conocimiento, el predominio de una visión positivista, estructural, funcionalista, y de una concepción instrumental del conocimiento, ${ }^{5}$ transformación que se encuentra con el

5. Creemos que con la proliferación de la universidad como recinto privilegiado de la transmisión del conocimiento a partir del siglo XIX se procuró la inserción de los estudiantes en la cultura del saber formalizado, alejándose de la concepción del pensamiento como arte de dar forma conceptual a las experiencias humanas. En nombre de la crítica y contra el dogmatismo, el Renacimiento y el periodo moderno de la historia occidental significaron los esfuerzos por secularizar el mundo del saber y las investigaciones, la construcción sistemática y validada de conocimiento, gracias al esfuerzo del sujeto del pensamiento; esta sería la rebelión de los filósofos contra la perspectiva teológica (Wallerstein, 1997, pp. 9-12). Entonces se planteó una teoría con base en el supuesto de un orden natural de la vida social (Wallerstein, 2007, p. 14); sin embargo, la experiencia de la crítica habría de considerarse privilegio de los expertos en las ciencias, desconociendo con ello otras formas de pensamiento, otros saberes, siendo esta una forma de excluir y negar a los sujetos que hacen la historia, a aquellos cuyo pensar no responde al sentido de lo instrumental como principal y único objetivo. 
pasado colonialista y racista de las ciencias sociales. ${ }^{6}$ Entonces, los esfuerzos disciplinares, que trataron de sistematizar campos de saberes que remiten a experiencias de sujetos y sociedades sustantivamente distintas, dieron lugar a la razón poscolonial y un saber neocolonial (Spivak, 2010, pp. 7-11), bajo el cual se posibilita la extensión del dominio, merced a la generalización de la economía capitalista, la política y la epistemología liberal, en nombre de los otros.

En un sentido semejante, Michel Onfray (2007, pp. 19-21) señala el predominio en la historia filosófica del idealismo, desde Platón hasta Kant y Hegel, que los libros de historia del pensamiento reproducen convertidos en instrumentos para continuar la imposición de dicha perspectiva. No obstante, esto no significa que no haya otras formas de pensamiento, entre ellas la que reconoce al sujeto como bio-psicosocial-histórico y totalidad singular; en todo caso, significa que tales pensamientos han sido excluidos y descalificados por varias vías. Una de ellas es la no publicación y difusión de las ideas, es decir, su marginación u omisión en la circulación de las ideas a través de los libros de historia del pensamiento y en los programas de estudio, haciendo del espacio universitario, antes que un promotor del pen-

6. De acuerdo con Gayatri Chakravorty Spivak (2010, pp. 13-117), el pasado colonial y racista de las ciencia sociales lo encontramos, por ejemplo, en la filosofía, en figuras del pensamiento como Kant, Hegel y Marx, quienes de distintas maneras representan la fuente de la formación del sujeto ético-político propiamente capitalista: el primero hablando de sujetos cuya minoría de edad no les permitía entender el mundo y la historia, además de que repudiaba al nativo; el segundo conceptuando a los no europeos y sus culturas como desviaciones de la razón histórica; $y$, en el caso del marxismo y de Marx institucionalizando la idea de los otros (los oprimidos y explotados del mundo) como sujetos de la revolución en boca de una vanguardia. La antropología por antonomasia expresa esa colonialidad, pues persiste hasta hoy, aunque con matices, la idea de que se trata del estudio de las otras culturas, las prácticas "singulares" de las comunidades. Su categoría ordenadora, etnia, habla por sí misma, pues con ella se piensa y nombra a la pluralidad de comunidades y pueblos no modernizados, cuyo pasado cultural es de larga data y su pasado inmediato no se vio transformado por ciertos procesos. 
samiento lúcido, una fuente de obstáculos para el pensar, en especial el pensar crítico.

La institucionalización del pensamiento como conocimiento validado por una instancia universitaria ha derivado, en la práctica académica, en la búsqueda del prestigio disciplinar como parte de un reclamo de los expertos para sí mismos. ${ }^{7}$ En este marco, nos interrogamos acerca de la pertinencia de permanecer en la universidad cuando reivindicamos un pensar epistémico y político para la creación de un mundo humano otro, más allá del capital y el Estado, en este caso también más allá de la instancia universitaria, interrogación que se articula con otra de relevancia y pertinencia ético-científica: ¿cómo podemos dejar de reproducir desde la dominación y crear otro mundo diferente?, y otra de carácter metodológico derivada de las anteriores: ¿cómo se plantea la necesidad de explorar nuevas formas de construcción del conocimiento?

Uno de los síntomas del deterioro y la implosión de la institución universitaria lo encontramos en la sobrestimación y la exigencia de renovación del conocimiento al ritmo del "progreso" (Nietzsche, 2010, p. 21). La implosión de las instancias de educación estaría en su pretensión de adecuarse a las exigencias del mercado. No obstante, cuando lo han logrado, esas exigencias ya han cambiado o ya ni siquiera existen (Castoriadis, 1996, p. 14). La versión más reciente de tal adecuación la encontramos en aquellos métodos enfocados al desarrollo de las habilidades y competencias, donde se anuncia como prioridad de los modelos educativos no los contenidos ni enseñar a pensar, sino que sepan "usar

7. En relación con identificar como expertos a quienes están en posesión de una parcela de conocimiento, pensamos, ello no tendría por qué suponer la subalternidad de otras formas de saber; sin embargo, está presente una tendencia a la instrumentalización de la expertis y de lo científico para neutralizar y descalificar la crítica emanada de otras posiciones y visiones en torno de los saberes y conocimientos socialmente necesarios para el fortalecimiento de procesos sociales de emancipación.

Teoría y DEBATE No. 66 
herramientas" facilitadoras de sus tareas, a lo que subyace el desprecio encubierto por la historia y la cultura propias; un ejemplo atroz es la tecnología incorporada como recurso didáctico, tornando precaria la relación en el aula, ya que ni siquiera se cuenta con las condiciones para ello.

Con la flexibilización y precarización del espacio universitario, emerge un tipo antropológico individuo-competencia solitario, provisto de tecnología, veloz, flexible, listo para el trabajo enajenado, egoísta y traicionero (Kurz, 2002a), en un contexto en el cual la sociedad es pensada como medio, productor y consumidor de todo tipo de mercancías. No perdamos de vista que en la sociedad capitalista del siglo XXI se impone la lógica de la mercancía. De acuerdo con Anselm Jappe (2009), en ella ya no se trata de un poder disciplinario que se despliega; la inteligencia académica ya no se necesita; la tendencia a la deshumanización con el capitalismo actual es posible gracias a la subjetivación reificada, ${ }^{8}$ a la socialización de sujetos que ven en el mundo medios para sus fines, y todo es sometido a los criterios del valor de cambio, instituyendo el fetichismo de la mercancía y su consumo, consecuente con la economía de financiarización. En tal marco, al mismo tiempo que se eliminan realidad, imaginación y horizontes de sentido, se impone una eterna adolescencia embrutecida, la barbarie cotidiana.

Con las formas capitalistas, el sujeto es la mercancía, sujeto que a los otros despoja de sus cualidades propias, y a cambio los torna abstractos (Jappe, 1998). Las personas, entonces, pueden ser flexibilizadas, es decir, explotadas, solitarias e insolidarias, cada vez más desconcentradas, para quienes el aprendizaje es mero consumo de un saber (Kurz, 2002). La industria pop es la nueva pedagogía: todo

8. No pasemos por alto que una subjetividad reificada es la reducción de un sujeto a la condición de medio para un fin, donde las relaciones, prácticas y procesos de los que forma parte serán vistos como algo ajeno, externo o que lo desbordan. Acuñamos la idea inspirados en el uso que Theodor Adorno hace del término. 
tiene que ser divertido, entretenido, nada complicado, y las escuelas y universidades en América Latina se alistan a actualizarse para cumplir con las exigencias de la economización y comercialización de la educación. Gobiernos, partidos, directivas universitarias, gremios académicos, disponen del poder para reducir la instrucción escolar a su mínima expresión formativa y su máxima rentabilidad. ${ }^{9}$ Ante este panorama, el espacio universitario se vislumbra como un cementerio de la creación cultural, porque se instituye con base en el absurdo mercado de los sujetos sin cualidades, de acuerdo con Anselm Jappe (2009).

En otros términos, se vive un proceso en el que la fuerza de trabajo y su naturaleza productiva han cambiado: ahora nuestro trabajo tiende a ser cada vez más inmaterial e impersonal, o bien sustituido por la tecnología y los sistemas en redes de comunicación. En esa nueva forma de explotación y dominación del trabajo también surgen formas de resistencia y voluntad colectiva. A través de la apropiación del conocimiento y el lenguaje de los sistemas de comunicación, los trabajadores podemos inhibir y desarticular el proceso de deterioro de la vida e iniciar la recuperación de nuestra libertad, para desde ahí luchar por un nuevo tiempo de vida, con justicia y dignidad, de acuerdo con los zapatistas del Ejercito Zapatista de Liberación Nacional.

Investigar y formarse más allá de la racionalidad capitalista

En la valoración anterior, partimos de observar que cuando un pensamiento es crítico está siempre en crisis y en rebelión, porque significa la posibilidad de rechazar

9. Los programas de licenciatura en el área de Ciencias Sociales y Humanidades de la Universidad de Guadalajara tienen como formación básica y obligatoria el curso Ética y gestión de la práctica profesional, enfocado a ofrecer a los estudiantes la idea de que hay una ética deontológica que deben conocer para integrarse creativamente en el mercado laboral.

Teoría y DEBATE No. 66 
participar de la extensión del dominio a través del saber disciplinario. En cambio, observamos con respecto al pensar teórico la tendencia a cerrarse sobre sus propios planteamientos. Al igual que los aparatos represivos y los aparatos del consenso, la educación escolarizada es utilizada para inhibir el arraigo del pensar epistémico y político de la creación humana y de la autonomía como proyecto, lo que contribuye a ahondar la crisis y la crítica de las relaciones sociales de dominio.

Los modos de apropiación de la materia de nuestro trabajo, y las formas de hacer política que hemos experimentado como profesores e investigadores, tienen en la autocrítica un punto de partida para reconocernos en el proceso de investigar, difundir, aprender, de manera que podamos contribuir a detener y revertir las políticas estatales de privatización de la educación, las condiciones de su flexibilización y el despojo de nuestro trabajo. Esto nos ha implicado dar cuenta del discurso manifiesto que los investigadores presentamos sobre el proceso y la materia de trabajo, además de las maneras de hacer(lo). Damos por supuesto lo que esto significa para la desmitificación del trabajo de investigación en cuanto a las formas y reglas establecidas (muchas veces reglas de hecho) respecto de la relación entre sujetos que estudian e investigan, para asumir que somos un sujeto entre una pluralidad de sujetos singulares y colectivos, que constituimos al sujeto social, y participamos del conocimiento socialmente construido.

En este sentido, y aceptando que las limitaciones de cada quien resultan de nuestra propia subjetividad, aun así, a esta la conceptuamos como subjetividad reflexiva y deliberante. ${ }^{10} \mathrm{~A}$ esto hay que agregar que las premisas epistémicas subyacentes en el proceso investigativo regu-

10. Pensar en la subjetividad reflexiva y deliberante es pensar en el sujeto como totalidad paradójica que alude a la potencialidad para pensar y reflexionar acerca de su constitución como dicho sujeto, con capacidad de reconocer la pertinencia 
larmente propician el ocultamiento del sujeto implicado en el problema de investigación. El tema es amplio y complejo. Baste señalar que pensamos en aquellas premisas y modos de indagar en los que, en favor del control de los datos y para sustentar las afirmaciones acerca de los procesos estudiados, se reducen a mediciones lo sociológico, lo histórico-cultural, o cualquier otra dimensión; el sujeto es parcelado en su complejidad hasta desaparecerlo, merced a su fragmentación en múltiples partes.

Así, en la interpretación de la realidad, aquella de la que se busca dar cuenta, cuando se recurre a estrategias metodológicas que utilizan la observación etnográfica, el análisis situacional, la investigación-acción, historias de vidas, genealogías, estudios de redes sociales, análisis del discurso, etnoencuestas, etc., no necesariamente estas pasan por una vigilancia epistémica que impida la negación del sujeto. ${ }^{11}$ Aún más, en muchos casos, el investigador no reconoce que es un sujeto, entre otros más, en el proceso de producción de conocimiento. En este sentido, uno de los aspectos relevantes a los que hemos llegado es la pertinencia de circunscribir y concretar la investigación en una postura epistémica que hemos llamado desde la perspectiva del sujeto a conocer. Esto significa no junto con el sujeto, ni sobre el sujeto, cuestión que consideramos se torna un

del ejercicio de su autonomía; esto es, aquel que reflexiona su propia práctica, de la que se desprenden las decisiones en relación con la orientación de su acción social y política. El sujeto es subjetividad reflexiva, capaz de deliberación y acción intencionada (Castoriadis, 1993, pp. 93-94).

I I. Desde nuestra perspectiva, la noción de interpretación en el psicoanálisis es la que mejor expresa la forma de hacer lo propio en relación con los sujetos de los movimientos de resistencia, pues se encuentra en el centro de la observación y escucha del trabajo psicoanalítico, y consiste en evidenciar el sentido latente de una representación discursiva o acción. A partir del relato del sujeto, e identificando resistencias y transferencias acerca de lo dicho, lo hecho, lo deseado, fantaseado, vivido y reprimido, es posible reconocer la significación que el sujeto atribuye; este proceso exige se logre una coincidencia entre sujetos involucrados en la interpretación (Laplanche y Pontalis, 2004, pp. 201-202). 
problema central epistémico-metodológico de la práctica investigativa. ${ }^{12}$

Retomamos del subcomandante insurgente Marcos (2003, pp. 4-5) la idea de la perspectiva del sujeto, quien, sin establecer una tipología, plantea las posturas productoras de conocimiento sobre los movimientos, en, con y desde los movimientos sociales y políticos, así como las interrogantes de para qué y contra qué se produce conocimiento. Tales diferencias, consideradas por sus implicaciones, evidencian que al problema de la teoría le corresponde también el de quién la produce, y permiten indicar que el uso de las teorías tiene consecuencias respecto de la práctica y viceversa. Entonces, señalan los zapatistas que su metateoría es su práctica; nos muestran una reflexión no acerca de sí mismos, sino de la realidad en la que se mueven, como la han vivido, como la enfrentan y quieren cambiar. El subcomandante insurgente Marcos (2003) dice: "al señalar y analizar, al discutir y polemizar, no sólo lo hacemos para saber qué ocurre y entenderlo, sino también, y sobre todo, para tratar de transformarlo" (pp. 4-5); por ende, generar reflexión teórica desde un movimiento social o político permite mostrar su práctica transformadora, los modos en que otros sujetos e iniciativas inician, siguen, acompañan, encuentran y abren espacios para sí mismos (subcomandante insurgente Marcos, 2003, p. 5). Se afirma entonces la relevancia del sujeto, el sentido y la dirección de su caminar transformador.

Ante esta distinción entre formas de generar saber, nos planteamos que

[...] [l]a problemática epistémica y ética en la producción del conocimiento desde la perspectiva del sujeto... [tiene como] referente empí-

12. Un rastreo acerca de las aproximaciones epistémico-metodológicas con que se ha abordado al sujeto, registradas en las revistas de antropología latinoamericanas, puede consultarse en Sujeto social y Antropología. Despliegue de subjetividad como realidad y conocimiento, de Jorge Alonso y Rafael Sandoval (2008).

\section{0}


rico el reconocimiento de la realidad de sujetos [...], la subjetividad desplegada en las interacciones intersubjetivas e intrasubjetivas, en situaciones concretas, en un dado-dándose que deviene en el hacer del sujeto situado en su contexto (Sandoval, 2012, p. 32).

Por tanto, pensar e investigar desde la perspectiva del sujeto $^{13}$ nos ha supuesto debatir acerca de

[...] la imposibilidad de reflexionar sobre el sujeto social otro, porque una cosa es la reflexividad que hace el sujeto de su propia práctica, en un ejercicio de autonomía, y otra cosa diferente es que otro sujeto realice una investigación sobre la práctica política, cotidiana, etc., de un sujeto social del que no es parte [...]. En este caso, lo que se pone en juego es la relación entre dos sujetos sociales diferentes, ello significa que la intersubjetividad está condicionada por la transferencia y la contratransferencia entre los sujetos, es decir, el factor subjetivo de lo psíquico y lo histórico-social que son constituyentes del sujeto que pretende conocer a otro sujeto, sin que necesariamente se correspondan en la interpretación (Sandoval, 2012, p. 34).

Por otro lado, están los procesos investigativos de académicos en los que se estudian los movimientos sociales desde la teoría, cuyo trabajo difícilmente supera el intento de adaptar la realidad a la teoría desde la cual están mirando el movimiento. El problema es que el flujo social del hacer de los sujetos no es algo que pueda ser observado, calculado ni diagnosticado en sus posibilidades de concreción, a menos que se sea integrante de los sujetos que lo generan

13. La perspectiva del sujeto, con base en un explícito posicionamiento político, se experimentó en el desarrollo de la investigación doctoral respecto de las formas de hacer política que niegan la racionalidad capitalista, y las que niegan al sujeto social, para enfatizar la pertinencia de reconocer, en el despliegue de la subjetividad, la capacidad de autorreflexividad crítica del sujeto para crear su propio conocimiento (véase: Sandoval Álvarez, R. (2007). Más allá de la racionalidad capitalista. Nuevas formas de hacer política (tesis doctoral inédita). Benemérita Universidad Autónoma de Puebla: Puebla).

Teoría y DEBATE No. 66 
(Sandoval, 2012, p. 39), ello debido a que existe una asimetría en la relación de conocimiento entre sujetos diferentes, correspondiente a cosmovisiones distintas, asimetría que trae consigo la intraducibilidad lingüística y cultural. ${ }^{14}$

Pensar más allá de la racionalidad instrumental conlleva colocarse $^{15}$ de tal modo que el saber en cuanto poder se diluya, pues la relación de trabajo de investigación se trastoca cuando, por ejemplo, la conversación dialógica se torna en un instrumento por parte del investigador académico con pretensiones de ser asesor, concientizador, capacitador, sistematizador, facilitador, o cualquier función que suplante al sujeto en su quehacer autónomo.

Por otro lado, advertimos la crítica de los sujetos sociales hacia la labor de investigación en el no requerimiento de hermeneutas que los expliquen, porque se trata de respetar al sujeto, sus formas de hacer, sus ritmos y temporalidad. Esto es, en el ámbito del conocimiento, cuando significa construcción social de sujetos, hablamos también de construcción de lo colectivo y no sólo construcción social de conocimiento. Para nosotros, en los procesos de conocimiento, la forma de compartir debiera adquirir carácter de principio epistémico y ético-político.

Por otro lado, la sensibilidad epistémica en vinculación con la postura del presente potencial también ha sido alternativa de la perspectiva del sujeto. En un momento deter-

14. La intraductibilidad lingüístico-cultural cuando se plantea una relación asimétrica entre el sujeto investigador y el sujeto investigado ha sido propuesta por Silvia Rivera Cusicanqui (2007). Véase: "El potencial epistemológico y teórico de la historia oral: de la lógica instrumental a la descolonización” (en Voces recobradas. Revista de Historia Oral, 8(2I), I2-22).

I5. La idea de colocación se refiere a la acción de conectarse, de situarse desde el contexto histórico, cultural, temporal-espacial de una realidad social o problema específico. Se trata de establecer una relación de conocimiento no encerrada en atributos. Para Zemelman (2000, p. 32), colocarse supone liberarse de parámetros y formas de razonamiento científico que presionan al conocimiento hacia formas ahistóricas y reduccionistas; se trata de recuperar la exigencia de mirar la realidad antes de volcarse en su explicación.

\section{2}


minado, se tornó en una opción al percatarnos de que, si de dar cuenta del sujeto y su perspectiva se trata, entonces no tiene como posibilidad exclusiva ser parte de un colectivo que lo constituya, tampoco supeditarse al mismo, sino que en la apertura de una ventana a la reflexividad y el hacer sociopolítico del sujeto media una participación afectivointelectual en las expectativas, problemas y necesidades de este. Ello significó poner en común la pretensión de autonomía para así ponderar su subjetividad y explicitar lo comprendido y valorado del sujeto, este entendido como posicionado, reflexivo y fuente de proyectos singulares de autonomía. ${ }^{16}$

Estas posturas surgen de observar que en las investigaciones y estudios con frecuencia el sujeto de estudio se encubre, se oculta o se niega, aunado esto a la dificultad de plantear un problema de la investigación. ${ }^{17}$ De acuerdo con Hugo Zemelman (2000, p. 217), la comprensión de la realidad se convierte en un obstáculo cuando no somos capaces de reconocerla como problemática, y ni siquiera la experiencia ni la teoría nos permiten acceder a ella. Si rompemos ese parámetro entonces nos damos cuenta de que hay algo nuevo en la captación del mundo.

Con Zemelman, encontramos como recurso epistemológico la delimitación del problema eje, tomado en tanto punto

16. La alternativa de la sensibilidad epistémica con base en un explícito posicionamiento político se experimentó en el desarrollo de la investigación doctoral acerca del proyecto de autonomía encarnado por dos colectivos mexicanos: la Brigada Callejera de Apoyo a la Mujer "Elisa Martínez", y el Colectivo Rebelión Cotidiana (véase: Salcido, R. (20l3). El trabajo a favor de la autonomía, elaboración de experiencia y afirmación anticapitalista (tesis doctoral inédita). Universidad de Guadalajara: Guadalajara).

17. Cuando el sujeto es encubierto o negado, se le sustituye por descripciones historiográficas o etnográficas con las que no se da cuenta del sujeto subyacente a las fuentes o a los discursos manifiestos, de manera que se le constriñe a una dimensión, sin contemplar todas las demás que constituyen al sujeto de estudio y que son necesarias para dar cuenta de la complejidad del hacer histórico-social del sujeto.

Teoría y DEBATE $\&$ No. 66 
de partida en la reconstrucción articulada de la realidad ${ }^{18}$ y en la tarea de dar cuenta del vínculo entre el sujeto y su praxis. La problematización consiste en el planteamiento de interrogantes con el propósito de hacer la crítica al problema para decantar relaciones posibles (Zemelman, 2000, pp. 67-69). Este ejercicio necesita de la reflexividad crítica para entender la realidad como una totalidad concreta significada desde la experiencia de los propios sujetos.

Llegamos así al posicionamiento ético y epistémico del conocimiento como construcción desde diferentes saberes, donde la ciencia sólo es uno de ellos, pues con independencia de las disciplinas y el despliegue institucional del saber, existen saberes que devienen de cosmovisiones de pueblos y culturas diferentes de las que surgen otras matrices epistémicas. Creemos, pues, en la generación de conocimiento con base en cierta conciencia de la época y en tensión con las disciplinas. Por tanto, lo pensamos como un modo de establecer una relación con el momento histórico, el contexto, los problemas presentes en la sociedad. Generar conocimiento en tensión con las disciplinas, consideramos, no puede realizarse sino tomando una postura ético-política de carácter anticapitalista, siendo posible para nosotros asumir el propósito de transgredir y desbordar los propios prejuicios epistémicos y políticos, potenciar la apertura del sujeto desde las necesidades propias y emplazar la transformación del mismo; esta es una de las formas que puede adquirir el pensamiento crítico.

Por otro lado, el pensamiento crítico siempre ha estado en crisis y haciendo crisis respecto del pensar teórico, de manera que el despliegue del pensar epistémico y político

18. La Realidad, como totalidad, es el presupuesto de la reconstrucción articulada de la realidad. Esta consiste en un procedimiento epistémico-metodológico en el cual son delimitados los campos de observación correspondientes con los procesos económico, social, político, histórico, antropológico, cultural, psíquico, los cuales son considerados resultado del hacer de los sujetos; por tanto, la delimitación ha de realizarse de acuerdo con estos.

\section{4}


en perspectiva de la creación humana y la autonomía como proyecto es otra forma de hacer desde la resistencia anticapitalista. Señalar la permanente crisis nos permite aludir al reacomodo epistémico al que se sujeta el pensar crítico para responder ante la incesante transformación de la realidad, puesto que una crisis representa posibilidades susceptibles de decantarse en una indeterminada dirección con base en la decisión y acción de los sujetos. Esto significa que participamos de la crisis del pensamiento al distinguir y optar por el punto de partida anticapitalista con el propósito de enfrentar la tarea de elaborar conocimiento en la perspectiva del sujeto de la autonomía, a partir de ensayar un pensamiento cuya búsqueda desemboca en la creación de formas conceptuales que permiten problematizar lo dado y dar cuenta de relaciones de posibles.

El trabajo académico universitario también puede significar una posibilidad de crear espacios autónomos, en la medida en que, como parte de nuestro trabajo cotidiano, se generen iniciativas más allá de los límites del currículo establecido, o bien, promoviendo otra forma de relación entre sujetos en el proceso de investigación; en torno de este punto es que hemos discutido y elaborado nuestras propuestas de investigación desde hace más de una década.

Dos aspectos nos resultan significativos, a propósito de la potenciación de nuestro trabajo en la universidad: uno es que se trabaja no para la institución, sino respecto de lo que consideramos son las necesidades presentes en el entramado social; el otro es fomentar el encuentro con quienes asumen preocupaciones similares. Como trabajadores docentes e investigadores en una universidad pública mexicana, esa no-mentalidad de funcionario la hemos trabajado, asumiendo una posición antagónica respecto de lo que se propone como modelo educativo, y posibilitando habitar la contradicción que nos representa participar desde la docencia e investigación en la generación y socialización del 
conocimiento. Lo hemos procurado a partir de no separar la docencia y la investigación, y estas en relación con los problemas de nuestro contexto. ${ }^{19}$

En el contexto latinoamericano, este tema complejo está vinculado con la relación del intelectual con un compromiso más allá de lo propiamente académico (Zemelman, 2012), porque en nuestros países tiene que ver con la burocratización del hacer investigativo y la parcelación del conocimiento por “especialidades". Afirma Zemelman, a propósito del intelectual:

[...] es una persona que no tiene trascendencia histórica, fue un hombre débil, un hombre sometido, es un pusilánime, es cobarde; claro, todo esto disfrazado con grandes parafernalias. Un hombre no comprometido ni siquiera con lo que dice, $y$ por tanto, con una gran predisponibilidad de desarrollar una mentalidad de burócrata [...], han terminado en transformarse en funcionarios: calificados, honestos, pueden ser hasta creativos, pueden ser muy responsables, no son juicios morales, son más bien situaciones estructurales [...]. También es un tema interesantísimo el considerar que el intelectual perdió la capacidad de tener proyectos y se transformó simplemente en un eficiente experto, digamos, de funciones precisas, pero sin proyectos -cuando hablo de proyectos estoy hablando, no de proyectos puramente normativos, estoy hablando de proyectos intelectuales que sean también proyectos de vida-; o sea, se disoció el proyecto de vida del trabajo; y entonces, al disociarse el proyecto de vida del trabajo, hay que estar bien en el trabajo porque el proyecto de vida supuestamente lo hago en otra parte, lo que es una gran mentira, porque tampoco se hace; $y$ entonces eso llevó, entre otras

19. En los esfuerzos por generar resonancias entre los contenidos de nuestros programas docentes, nuestros proyectos de investigación y las problemáticas sociales tienen un ejemplo en Cómo pensar la revolución hoy, proyecto que se enlaza con nuestro quehacer docente, pues se encuentran en el propósito de mostrar la necesidad de pensar en la lógica de lo por ser, del presente potencial, así como de hacer un uso crítico de los conceptos. De igual forma, la temática de este proyecto es la concepción de transformación social gestada por colectivos que son parte del movimiento de resistencia anticapitalista.

\section{6}


múltiples cosas, a que sean profundamente inconsistentes, acomodaticios, balbuceantes en las cosas importantes, y gritones y chillones en los menos importantes. Llevó en definitiva como gran tema filosófico y epistémico al divorcio con el discurso; un discurso sin sujeto, o sujetos con un discurso en el que no creen, pero que les sirve como ropaje (Zemelman, 20I2).

Para responder a las interrogantes que nos planteamos al inicio, pensamos, como elemento primario, no desplegar la materia de trabajo con una mentalidad de funcionario y potenciar la experiencia de construcción y socialización del conocimiento; la experiencia de diseñar, como académicos, un proyecto en el que se encuentren articuladas la propia vida y el trabajo. Porque vemos el problema en que se ha convertido el simulacro de labor y vida académica, y esto está presente en todo el espectro de espacios de formación y en los diferentes niveles de educación universitaria, así como vemos la reproducción de una actitud teoricista en el proceso de enseñanza-aprendizaje, cuyas implicaciones directas no son sólo teóricas, sino también políticas, al contribuir con ello a la reproducción de relaciones de dominación, aun cuando en muchos casos se manifiestan de acuerdo con un cambio de las relaciones sociales y políticas.

Resulta problemático enfrentar esta situación al estar de por medio la posibilidad de legitimidad que la institución universitaria requiere, disfrazada, en el ámbito del aula, de libertad de cátedra, pues es el lugar donde se concreta el consenso sobre las directrices en los planes de estudio, políticas de extensión y difusión, que van desde la política que decide qué, a quién y cómo se pública en libros, revistas, o quién se presenta en programas de televisión y radio, obras de teatro, cine, etc., hasta los términos en que se decide la conformación de cuerpos académicos, la integración de las plantillas de profesores en el pregrado y posgrado, sin que se tome efectivamente en cuenta la pluralidad de posiciones manifiesta en 
la base de profesores, investigadores, estudiantes, difusores y demás trabajadores de la educación; ello expresa en la cotidianeidad la instrumentalización de los saberes.

La persistencia de tales condiciones nos ha conducido a preguntarnos por las posibilidades para dejar de reproducir la forma acumulativa, instrumental y teórica del conocimiento, desde el proceso de enseñanza aprendizaje, desde la cotidianidad de las aulas y de las tareas de investigación que realizamos los profesores investigadores universitarios. Esto nos ha impulsado a asumir el desafío de una nueva forma de hacer política y generar conocimiento, nombrada aquí resistencia anticapitalista y autonomía como proyecto, tanto desde las universidades como al margen de ellas. Esto es el horizonte con base en el cual orientamos nuestro hacer social.

\section{Creación de comunidad de investigadores y profesores universitarios}

En las últimas dos décadas, poco más, se ha deteriorado la vida académica en lo sindical, en la cotidianeidad laboral, pues las universidades no fueron ajenas a las políticas de austeridad, contención salarial, flexibilización del trabajo, congelación de plazas y cooptación de trabajadores investigadores para desempeñar funciones de dirección administrativa. La problemática que no terminamos de enfrentar en lo general es parecida a la que viven la mayoría de las organizaciones de trabajadores: no hemos sabido destruir el corporativismo paternalista y clientelar. ${ }^{20}$

20. Un ejemplo del corporativismo es la corrupción y manipulación que favorecen las directrices políticas y laborales de cooptación y fragmentación del sujeto universitario que desde las instancias universitarias se ha implementado, de modo que se ha logrado que los propios investigadores y profesores, al acceder a puestos de dirección, jefaturas departamentales y administradores de los centros investigación y coordinadores de carrera, así como los miembros de los consejos universitarios, permitan y avalen la destrucción de la universidad y la educación pública y gratuita. 
En tal contexto, reconocemos que no hemos experimentado nuevas formas de hacer en política, como tampoco en la docencia y en la investigación. Por ello, planteamos la autonomía en el sentido de que quienes laboramos en la universidad, investigadores y profesores, determinamos el proceso de trabajo, a través de la apropiación de nuestra materia de trabajo desde la cotidianidad de las aulas.

En este sentido, creemos que el autocontrol que conlleva el infrapoder capitalista es más decisivo que cualquier aparato de dominación institucional. ${ }^{21}$ Gracias a este es que las políticas laborales del Estado han logrado fragmentar a los universitarios, ya que favorece un ambiente de relaciones personales entre los académicos que raya en una descarnada y disimulada competencia individual; la envidia y la traición en asuntos laborales también son producto del sistema de competencias que se ha impuesto. ${ }^{22}$

Dadas estas circunstancias, estamos obligados a enfrascarnos en un proceso de reapropiación y defensa de nuestra materia de trabajo, y buscar que seamos los trabajadores quienes ejerzamos autónomamente las instancias técnicas y organizativas del trabajo investigativo y de formación.

Hacer de nosotros mismos sujetos del proceso de trabajo es parte de la lucha contra la destrucción de la educación pública; ello exige también una forma de hacer política que la articule con la lucha de los pueblos, comunidades

2I. El infrapoder es propio de lo imaginario instituyente, de la sociedad instituida y de la historia, es el poder del campo social-histórico, el cual no puede prescindir del poder explícito (este es lo que se acostumbra nombrar como poder político), ya que aparece como la satisfacción de la necesidad de decisión respecto de lo que debe o no hacerse en relación con los fines sustantivos de la sociedad (Castoriadis, 2000, pp. 52 y 56), siendo estos relativos al proyecto de la sociedad; el infrapoder es la condición propicia para la fabricación social de los individuos y es compensador del orden/caos de la vida colectiva.

22. En relación con la determinación de las políticas internacionales en educación de los modelos educativos en México, en la Universidad de Guadalajara realizamos una ponencia (Gallegos, Sandoval y Salcido, 2002) en la cual nos propusimos dar cuenta del desfase entre las políticas institucionales y las necesidades y expectativas sociales en relación con la generación de conocimiento.

Teoría y DEBATE $\&$ No. 66 
y barrios. Por ello, está pendiente de entenderse que la defensa de la educación implica, en la actual situación, enfrentar el despojo del territorio y la cultura, pues educación, historia, territorio, cultura, política, no pueden comprenderse como ámbitos independientes, a menos que desconozcamos a los sujetos que hacen posible la existencia de las instituciones de educación.

Desde la resistencia y la autonomía, se han generado iniciativas políticas y organizativas en las universidades que se concretan en la cotidianidad: la construcción de autonomía con base en proyectos, en la perspectiva de que sean los propios sujetos sociales quienes se hagan cargo de la reproducción de su vida digna, y con ello, de su aprendizaje. En nuestro país, México, en agosto de 2013, se dio una muestra de cómo decenas de miles de indígenas zapatistas están creando su educación autónoma. ${ }^{23}$

En este sentido, nos preguntamos, como investigadores y profesores universitarios, de qué manera hemos participado y contribuido en los procesos de autonomía, pero también cómo ha de ser la forma autonómica en el marco de la institución universitaria que permita la apropiación de la materia y el proceso de trabajo. Hemos estado ahí, de manera consciente o inconsciente, contribuyendo a su defensa o a su fetichización, ${ }^{24}$ mas con la pretensión de fomentar el surgimiento de la comunidad de aprendizaje.

23. En el mes de agosto de 2013, el Ejercito Zapatista de Liberación Nacional y las bases de apoyo abrieron una ventana a su proceso de autonomía a través de la iniciativa La Escuelita Zapatista. La libertad según los zapatistas. En este primer ciclo, nos permitieron ver la forma caracol de hacer política autónoma. Los temas expuestos fueron el Gobierno zapatista, el Gobierno de mujeres, la resistencia, la justicia autónoma y el modo zapatista de la democracia.

24. La conciencia de la necesidad de desfetichización significa reconocer en principio que estamos sometidos a la explotación de nuestro trabajo y a la alienación de la conciencia por medio de la educación que impone el sistema político-cultural, de manera que se manifieste la impronta en la necesidad de cuestionar y criticar esos procesos en la medida que se convierten en insoportables y que no resuelven las mínimas condiciones de reproducción de la vida digna. 
Pensamos que se trata de experimentar nuevas formas de hacer investigación y política, de romper con los hábitos de pensamiento perniciosos.

Así mismo, partimos de que una instancia pública como la universidad es innegablemente social, considerando que quienes las constituimos somos sujetos del proceso de formación, generación y transmisión de conocimiento, pues el aprendizaje es para nosotros una necesidad, de la cual faltaría precisar el sentido que tiene la misma. Por otro lado, las implicaciones que trae consigo la formación de seres humanos para la vida exigen una ética y una forma de hacer que permite mostrar quiénes somos y nuestra manera de participar del proceso instituyente del aprendizaje.

De ahí que una de las primeras cuestiones a tener en cuenta es el ejercicio de la autonomía, cuyas potencialidades pueden desplegarse desde lo que somos, estudiantes, profesores e investigadores, en tanto nos reconozcamos como tales y portadores de formas embrionarias de saberes y conocimientos. ${ }^{25}$

Una relación "enseñanza-aprendizaje" de carácter autónomo ha sido posible priorizando lo que en potencia somos, pero desde lo que hacemos. Tratamos de responsabilizarnos de la tarea de autoformación para ejercer una profesión, un oficio, una tarea, cuyo hacer impactará el tejido social: la generación de conocimiento pertinente, que significa, entre

25. No pasamos por alto que el conjunto de la vida universitaria, en tanto institución, también conlleva la administración. Esta dimensión operativa que conduce la vida burocrática es realizada por la mediación de los trabajadores administrativos y de servicio, aspecto que afecta sólo colateralmente, en los términos que se están planteando, la idea de la comunidad de aprendizaje, la relación sustancial entre profesores y estudiantes, así como la realización de investigaciones. Abordar esta cuestión en sí misma implica problemáticas específicas si pensamos desde los sujetos de la administración universitaria, ya que la actividad de los sujetos del proceso administrativo y de servicio, así como el ámbito que conforman, suele ser demeritado, tanto como no valorado; cabe aclarar que pensamos ante todo en los trabajadores administrativos y de servicio de base.

Teoría y DEBATE $\&$ No. 66 
otras cosas, la atención a las demandas de conocimiento, la transmisión de conocimiento y la "socialización" de formas de percibir y reflexionar la realidad, de la que somos parte.

Esto demanda de nosotros la apropiación de modos de comprender, de conocer, la interiorización de formas conceptuales, y los modos de usarlas críticamente, como sujetos de una comunidad que, aunque enajenada en su conjunto, presenta despliegues y manifestaciones emergentes de resistencia a la institucionalidad alienante respecto de la necesidad de hacernos partícipes y recíprocamente activos en la relación enseñanza-aprendizaje-producción de conocimiento.

Esta ha sido nuestra manera de iniciar la restitución de nuestros haceres como haceres críticos en perspectiva de la apropiación de la materia de trabajo y el proceso de producción de conocimiento, en función de satisfacer la necesidad de conocimiento y de formación. Ha sido nuestra manera de empezar a negar la negación de que hemos sido objeto por la fetichización de nuestra práctica, esto a través de despojarla del carácter instituyente y creador que, en forma embrionaria, se despliega a través de nuestras críticas; también, a través de los ejercicios de reflexión al margen de los modelos educativos impuestos; y a través de compartir preocupaciones con otros miembros de la comunidad universitaria, etcétera. Esta ha sido nuestra manera de enfrentar la pregunta de cómo ejercemos la autonomía como proyecto, al tiempo que dejamos de reproducir la forma en la que uno sabe y otro no sabe, uno enseña y el otro aprende.

También hay que decir que quienes aspiramos a instituir la comunidad de aprendizaje no buscamos posicionarnos en la estructura universitaria, menos si implica subordinación y dominación, porque se trata de dejar de hacer la institución como parte del sistema de educación capitalista, donde aprender significa hacerlo en función de los intereses de acumulación, de acuerdo con la exigencia del mercado y sus relaciones de producción. Entonces, experimentamos la 
comunidad de aprendizaje como un proceso de enseñanzaaprendizaje-investigación-circulación de las ideas, el cual, además de formarnos, nos permite lograr conciencia histórica, conciencia política y conciencia psíquica.

En este caso, por comunidad de aprendizaje no se entiende un grupo de personas constituido como tal, sino una fluctuante interacción entre sujetos que en principio tratamos de desaprender los hábitos instrumentales que emergen en la generación de conocimiento. De igual forma, tratamos de reconocer las preocupaciones y problemáticas de los sujetos en su contexto, de la población como sujetos (y no objetos) involucrados en la investigación, el estudio y la difusión de las ideas acerca de ellos. El autorreconocimiento nos ha significado hacer una radiografía de la situación y el contexto en que nos encontramos: realizamos un autodiagnóstico con base en el hacer social y la apropiación de nuestro poder hacer. ${ }^{26}$

Por ello, la comunidad de aprendizaje que tratamos de impulsar ha de surgir de que los profesores compartan su saber hacer, y que los estudiantes problematicen ese saber hacer para pensarlo distinto, y al mismo tiempo los profesores problematicen los replanteamientos de los estudiantes. Su integración por grupos ha de surgir a partir del interés en talleres, temas, proyectos, seminarios o actividades, en los que la prioridad sea emplazar al estudiante a investigar y pensar desde sí mismo, su contexto y lo que otros han hecho o dicho.

La comunidad de aprendizaje la pensamos, por ejemplo, como un ámbito en el que la evaluación no es individual,

26. La idea de la radiografía y el autodiagnóstico no se debe considerar como una forma de reconocernos desde fuera o en un sentido instrumental-utilitario, al estilo de los análisis que sirven para configurar la estrategia de lucha sin sujetos concretos, es decir, sin considerar que somos los propios sujetos que constituimos el espacio institucional quienes debemos autorreconocernos. La radiografía y el autodiagnóstico se tienen que ir revelando y realizando en el propio caminar-preguntando sobre el hacer que satisfaga nuestras necesidades como comunidad de aprendizaje. 
sino una valoración acerca del trabajo realizado en común. La expectativa es lograr que funcione, en la medida de lo posible, como colectividad organizada, con diferentes grados de involucramiento, donde logremos considerar el conocimiento en tanto consecuencia de la compartición de lecturas, miradas, reflexiones y experiencias.

Por tanto, la comunidad de aprendizaje nos ha significado problematizar nuestra forma de participar de los procesos universitarios en su conjunto, no sólo la propia labor, para tomar nuestro hacer como una cuestión tanto ético-política como pedagógica e investigativa. No podemos decir haber encontrado un modo de hacer comunidad de aprendizaje que nos resulte más apropiado, pero sí podemos decir que cada vez se hace manifiesta la necesidad de replantear la iniciativa conforme al acuerdo de la pluralidad de sujetos partícipes.

Esta ha sido nuestra manera de tornarnos sujetos del proceso de investigación y enseñanza-aprendizaje. En cuanto la posibilidad de formación para la realización de procesos que permitan a nuestra sociedad resolver los problemas para caminar en la perspectiva de una vida digna, sería conveniente empezar por reconocernos. Es así que nos hemos interrogado acerca de quiénes somos los estudiantes, profesores, investigadores, trabajadores en la universidad, qué hacemos y de qué manera lo hacemos. Es posible que la emergencia de formas de autonomía en el espacio universitario, a través de iniciativas de autoformación, permita pensarlo como espacio no exclusivo de las políticas capitalistas en educación y conocimiento, no obstante el carácter conservador que ha prevalecido.

\section{A modo de conclusión}

Señalamos que, en ese camino, el horizonte ético y las formas de hacer acordes con la construcción de autonomía tendrán que irse pensando y haciendo de acuerdo con prin- 
cipios que se han experimentado, tales como el mandarobedeciendo, que se opone a ejercer puestos de autoridad designados por los de arriba, ${ }^{27}$ el caminar-preguntando, que se opone a imponer una línea de acción desde fuera y desde arriba; y el para todos todo, que exige la inclusión de todos en la perspectiva de ensayar otras formas de hacer y de la relación social en la formación y la investigación.

De nuestra parte, ha significado empezar desde abajo y con los que vemos la necesidad de ser sujetos de nuestro propio proceso de formación y de conocimiento. Con base en la iniciativa de la comunidad de aprendizaje se pretende propiciar la participación de los distintos universitarios, estudiantes, docentes e investigadores, aun cuando no suscriben el horizonte de la autonomía, en las tareas de enseñanza-aprendizaje, de investigación y socialización de las ideas. No obstante, al estar de acuerdo en enfrentar las contradicciones y ambigüedades que como sujetos nos constituyen, también ha surgido la exigencia de ser consecuentes y coherentes con la pretensión de apropiación de la materia de trabajo.

De igual manera, en tanto determinamos que la comunidad de aprendizaje sea la mediación desde la cual nos apropiemos del proceso y la materia de trabajo, ello también significa que nuestra autonomía no surge por la estipulación de un principio, sino que vamos posibilitando nuestro hacer autónomo en la medida que reconocemos la autonomía de los otros con los que compartimos el espacio universitario.

Finalmente, en el trabajo de investigación y su encuentro con el trabajo docente, esto nos ha implicado enfatizar la crítica en relación con el pensamiento heredado, pero también respecto del uso crítico de los saberes, donde sujeto, formación, reflexividad, autonomía son, entre otros, los

27. Mandar obedeciendo, comunidad de consenso, caminar preguntando, entre otras categorías, están tomadas del discurso del Ejercito Zapatista de Liberación Nacional.

Teoría y DEBATE $\&$ No. 66 
conceptos centrales, así como recordar que todo pensamiento es una labor colectiva y de asunción de la perspectiva del pensamiento crítico como posibilidad de ruptura, siendo este un ejercicio de resonancia de la apuesta por la autonomía en el pensar generador de conocimiento y en las formas de hacer política.

Fecha de recepción: 14 de abril de 2015 Fecha de aceptación: 02 de febrero de 2016

Bibliografía

Alonso, J., y Sandoval, R. (20I2). "Sujeto social y Antropología. Despliegue de subjetividad como realidad y conocimiento", en P. González Casanova (coord.), Conceptos fundamentales de nuestro tiempo (p. 53). México: Universidad Nacional Autónoma de México, Instituto de Investigaciones Sociales. Recuperado de: http://conceptos.sociales.unam.mx/conceptos_final/480trabajo.pdf

Andrade, L. (2007). "Del tema al objeto de investigación en la propuesta epistemológica de Hugo Zemelman”. Cinta moebio, (30), 262-282.

Berger, P., y Luckmann, T. (1983). La construcción social de la realidad. Buenos Aires: Amorrortu.

Cornelius, C. (1993). "Psicoanálisis y política”, en, El mundo fragmentado (pp. 9I-I02). Uruguay: Nordam, Altamira.

_ (1996). "Conferencia pública: la crisis actual". Zona erógena. Revista Abierta de Psicoanálisis y Pensamiento Contemporáneo, (29). Recuperado de: https://n-I.cc/ file/download/ / 239083

- (2000). "Poder, política, autonomía”, en, Ciudadanos sin brújula (pp. 45-74). México: Coyoacán.

Gallegos, M., Salcido, R., y Sandoval, R. (2002). “Apuntes para una agenda de discusión sobre ciencia, cultura y educación superior en México". Ponencia presentada en el Foro sobre la Problemática de las Universidades 
Públicas. La política educativa y sus implicaciones, 25-27 de septiembre. Universidad de Guadalajara: México. Jappe, A. (3I de diciembre de 1998). "Las sutilezas metafísicas de la mercancía”. Krisis. Kritik der warengesellschaft. Recuperado de: http://www.krisis.org//998/las-sutilezas-metafisicas-de-la-mercancia

- (2009). "Introducción a la edición castellana", en R. Kurz, A. Jappe, y C. P. Ortlieb (auts.), El absurdo mercado de los hombres sin cualidades. Ensayos sobre el fetichismo de la mercancía (pp. 7-26). Logroño: Pepitas de Calabaza. (2002). "Epílogo. La persona flexible. Un carácter social nuevo en la sociedad global de crisis", en Grupos Krisis (auts.), Manifiesto contra el trabajo (pp. 73-79). Barcelona: Virus Editorial. Recuperado de: http://www. viruseditorial.net/pdf/Manifiesto\%20contra\%20el\%20 trabajo.pdf

( 10 de diciembre de 2002a). "La comercialización del alma”. Mensaje en blog publicado por Pimienta negra. Recuperado de http://br.groups.yahoo.com/group/ eskuerra/message/III 82

(2003). “La metamorfosis del imperialismo”, en, La guerra de ordenamiento mundial. El fin de la soberanía y las mutaciones del Imperialismo en la era de la globalización $(\mathrm{s} / \mathrm{p})$. Lisboa: Holermann Verlag. Recuperado de: ebeco. no.sapo.pt/rkurz_es I66.htm

Laplanche, J., y Pontalis, J. B. (2004). Diccionario de psicoanálisis. Barcelona: Labor.

Mancoksky, V. (2009). “¿Qué se espera de una tesis de doctorado? Breve introducción sobre algunas cuestiones y expectativas en torno a la formación doctoral”. Revista Argentina de Educación Superior, I (I), 20I-2I6. Recuperado de: http://www.untref.edu.ar/raes/documentos/ Que\%20se\%20espera\%20de\%20una\%20tesis\%20de\%20 doctorado.pdf 
Bibliografía
Nietzsche, F. (20I0). Sobre el porvenir de nuestras escuelas. México: Tusquets.

Onfray, M. (2007). "Preámbulo general. La historiografía. Un arte de la guerra”, en, Las sabidurías de la antigüedad. Contrahistoria de la filosofia. Tomo I (pp. 15-28). Barcelona: Anagrama.

Rivera Cusicanqui, S. (2007). "El potencial epistemológico y teórico de la historia oral: de la lógica instrumental a la descolonización". Voces recobradas. Revista de Historia oral, 8(2I), I2-22.

Sandoval, R. (2008). El zapatismo urbano de Guadalajara. Contradicciones y ambigüedades en la forma de hacer política. México: Instituto Nacional de Antropología e Historia. (20I2). Más allá de la racionalidad capitalista. Nuevas formas de hacer política. México: Instituto Nacional de Antropología e Historia.

Spivak, G. (20I0). Crítica de la razón poscolonial. Hacia una historia del presente evanescente. Madrid: Akal.

Subcomandante insurgente Marcos (2003). "El mundo: siete pensamientos en mayo de 2003”. Rebeldía, I (7), 3-I4.

Wallerstein, I. (1997). La historia de las ciencias sociales. México: Centro de Investigaciones Interdisciplinarias en Ciencias y Humanidades, Universidad Nacional Autónoma de México.

(2007). Abrir las ciencias sociales. México: Siglo XXI Editores, Centro de Investigaciones Interdisciplinarias en Ciencias y Humanidades, Universidad Nacional Autónoma de México.

Zemelman, H. (1990). "Los sujetos sociales, una propuesta de análisis". Acta Sociológica, III(2), 89-I04.

(1999). "La Historia se hace desde la cotidianeidad", en H. Dieterich (ed.), Fin del capitalismo global. El nuevo proyecto histórico (pp. 209-223). La Habana: Ciencias Sociales. 
(2000). Conocimiento y sujetos sociales. Contribución al Bibliografía estudio del presente. México: Colegio de México.

(I4 de abril de 2000a). "Método y sujetos sociales en América Latina”. Conferencia presentada en el Centro Universitario de los Altos. Universidad de Guadalajara: Tepatitlán, Jalisco.

(20II). Del "Chile inventado" y de otros tabúes. Hugo Zemelman, el pensamiento latinoamericano desde sus subjetividades (entrevista realizada por Erick Valenzuela). Recuperado de: http://www.lachispa.cl/201 I/06/del"chile-inventado"-y-de-otros-tabues-hugo-zemelmanel-pensamiento-latinoamericano-desde-sus-subjetividades

(2012). Seguir el camino, inventándose el camino (entrevista realizada por la Dra. Eugenia Trigo Aza). Popayán, Colombia: Universidad del Cauca. Recuperado de: http://www.consentido.unicauca.edu.co/site/DesplegarSeccion.php?seccion=Entrevistas/hugo\%20zemelman 